\title{
Unidade de Vigilância Intensiva COVID-19 do Hospital CUF Tejo, Fevereiro 2021: Memory Lane
}

\section{COVID-19 Intensive Surveillance Unit, CUF Tejo Hospital, February 2021: Memory Lane}

Cepeda Ribeiro ${ }^{1 *}$, Carolina Lalanda ${ }^{1}$, António Bugalho ${ }^{1}$

\section{RESUMO}

INTRODUÇÃO: No início de 2021, Portugal chegou ao infortúnio de ser o país com maior número de casos e mortes/100 000 habitantes por COVID-19 no mundo. Para permitir uma abordagem adequada a doentes hospitalizados com SARS-CoV-2 com diferentes graus de gravidade, o Hospital CUF Tejo procedeu a uma reorganização e criou uma unidade de vigilância intermédia (UVI).

MÉTODOS: Apresentamos o trabalho vivenciado na organização da UVI e a análise dos doentes assistidos. Consideraram-se critérios de internamento nesta unidade os doentes com pneumonia COVID-19 e necessidade de terapêuticas respiratórias não invasivas.

RESULTADOS: Entre 1 de fevereiro e 3 de março de 2021 foram seguidos 26 doentes, de um total de 28 internamentos, com predomínio do sexo masculino (69\%) e idade média de 76,8 anos. Dos doentes, 85\% apresentavam pelo menos uma comorbilidade. Em todos os casos o motivo de admissão foi a insuficiência respiratória, tendo sido iniciado oxigénio de alto fluxo e/ou ventilação não invasiva em 23 doentes. Foi instituída ou mantida corticoterapia em 24 doentes e antibioterapia em 22 doentes. A duração média do internamento na UVI foi de 6,2 dias. No total, 62\% tiveram alta hospitalar, 35\% faleceram e 1 encontrava-se internado à data de redação.

CONCLUSÃO: A plasticidade organizativa, a correta coordenação, a cooperação e articulação entre equipas, e o desenvolvimento de cuidados com base na melhor evidência científica disponível, foram elementos cruciais desde a génese da nossa atuação até ao momento em que a equipa deixou de exercer as funções, face à melhoria da situação pandémica COVID-19.

PALAVRAS-CHAVE: COVID-19; Insuficiência Respiratória; Oxigenoterapia; SARS-CoV-2; Ventilação não Invasiva 


\section{ABSTRACT}

INTRODUCTION: In early 2021, Portugal came to the misfortune of being the country with the highest number of cases and deaths/100000 inhabitants by COVID-19 in the world. To provide appropriate care to hospitalized patients with SARS-CoV-2 infection with different degrees of clinical severity, the CUF Tejo Hospital carried out an internal reorganization and set up an intermediate surveillance unit (UVI.

METHODS: We present the work experienced in the organization of the UVI and the analysis of the assisted patients. The admission criteria to this unit were patients with COVID-19 pneumonia and the need for non-invasive respiratory therapies.

RESULTS: Between February 1st and March 3rd, 2021, 26 patients were followed, out of a total of 28 admissions, with a predominance of man (69\%) and a mean age of 76.8 years. A total of $85 \%$ had at least one comorbidity. In all cases, the reason for admission was respiratory failure, with high-flow oxygen and/or non-invasive ventilation being initiated in 23 patients. Corticosteroid therapy was instituted or maintained in 24 patients and antibiotic therapy in 22 patients. The average UVI length of stay was 6.2 days. In total, $62 \%$ were discharged from hospital, $35 \%$ died and 1 was hospitalized at the time of writing.

CONCLUSION: The organizational plasticity, the correct coordination, cooperation and articulation between teams, and the development of care based on the best available scientific evidence, were crucial elements from the genesis of our work until the moment when the team ceased its functions, due to COVID-19 outbreak improvement of the COVID-19 pandemic situation.

KEYWORDS: COVID-19; Noninvasive Ventilation; Oxygen Inhalation Therapy; Respiratory Insufficiency; SARS-COV-2

\section{INTRODUÇÃO}

"A humanidade e os microrganismos patogénicos têm, desde sempre, mantido uma permanente competição: umas vezes ganhamos, outras perdemos."

Joshua Lederberg, Prémio Nobel da Medicina em 1958, especialista em biologia molecular

No início de 2021, Portugal chegou ao infortúnio de, estatisticamente, ser o país com mais casos e mortes/100 000 habitantes por COVID-19 no mundo.

A CUF - que desde o primeiro momento se organizou internamente para o combate nacional à pandemia, recebendo doentes que se dirigiam diretamente aos seus hospitais e foram transferidos pelo SNS ${ }^{1}$ - alargou a sua capacidade no tratamento de doentes COVID-19.

De forma a permitir uma capacidade de resposta aos doentes com necessidade de internamento e diferentes graus de gravidade clínica, o Hospital CUF Tejo procedeu a uma reorganização interna.

Perante a evolução da pandemia, a Administração decidiu criar uma Unidade de Vigilância Intermédia (UVI) com capacidade de 10 camas, totalmente dedicada a doentes COVID-19.?

Teve como objetivos dotar o hospital de uma estrutura intermédia diferenciada capaz de oferecer uma abordagem adequada a doentes que, não apresentando cri- térios de ventilação mecânica, exigissem uma vigilância intensiva e terapias respiratórias não invasivas (TRNI), impedindo a saturação da unidade de cuidados intensivos (UCIP), de forma a ter capacidade de resposta para os doentes mais graves que necessitassem de ventilação invasiva.

Em resposta à enorme pressão de internamento provocada pelos doentes que ao Hospital acorriam, a decisão foi rápida, exigindo uma célere adaptação das estruturas e profissionais, para fazer face ao desafio.

\section{ORGANIZAÇÃO}

A UVI foi estruturada em menos de uma semana.

Saímos da nossa área de conforto e metemos "mãos-à-obra" - "Because we have no other option, we will experiment, we will learn, and we will transform."3

Foram definidas a equipa médica residente, as equipas de enfermagem, fisioterapeutas e auxiliares de ação médica, bem como o modelo de funcionamento (escala de médicos residentes e não residentes para assegurar a permanência física de médico na UVI durante as 24 horas), e o adequado e necessário equipamento para uma unidade com estas características.

A unidade foi equipada com material de ventilação não invasiva (VNI) - CPAP (pressão positiva aérea contínua), 
CPAP com helmet, BIPAP (pressão positiva aérea bi-nível) -, oxigénio de alto fluxo por cânulas nasais (HFNO, high flow nasal oxygen), ventilador "de resgate", equipamento de monitorização contínua na sala de enfermagem e sala da equipa médica, material consumível e listagem de medicação.

As normas de segurança relativamente às condições ambientais da unidade e os equipamentos de proteção individual (EPI) foram igualmente uma das preocupações, definidas em conjunto com a equipa da PPCIRA (Programa de Prevenção e Controlo de Infeções e de Resistência aos Antimicrobianos) do hospital.

Os critérios de internamento e protocolos de atuação foram elaborados pela equipa médica residente, e no dia 1 de fevereiro foi formalmente aberta a UVI.

Consideraram-se critérios de internamento nesta unidade os doentes com pneumonia COVID-19 e necessidade de terapêuticas respiratórias não invasivas, nomeadamente a ventilação não invasiva (VNI) e/ou oxigénio de alto fluxo (HFNO). Os doentes com $\mathrm{SpO}_{2}<93 \%$ com $\mathrm{O}_{2}$ a $5 \mathrm{~L}$ ou $\mathrm{FiO}_{2} 40 \%$ preenchiam critérios de admissão na UVI.

Adotou-se como protocolo de atuação o algoritmo proposto num artigo de referência recentemente publicado por Winck JC et al, ${ }^{4}$ que foi revisitado e simplificado por João João Mendes, intensivista do Hospital CUF Tejo com experiência clínica acumulada no tratamento de doentes COVID-19 em unidade de cuidados intensivos desde a $1^{\text {a }}$ vaga.

Na tomada de decisões foi privilegiada a avaliação clínica, imagiológica e laboratorial, e os índices de $\mathrm{Rox}\left(\mathrm{SpO}_{2} /\right.$ $\left.\mathrm{FiO}_{2}: \mathrm{FR}\right)$ e de Horowitz $\left(\mathrm{PaO}_{2}: \mathrm{FiO}_{2}\right)$.

A terapêutica de suporte ventilatório (VNI e/ou HFNO), considerada essencial na insuficiência respiratória aguda induzida pelo SARS-CoV-2, foi associada a decúbito ventral como terapêutica complementar, sempre que tolerada.

Mais frequentemente foram implementadas as seguintes terapêuticas adicionais:

- corticoterapia sistémica de acordo com as Recomendações da Sociedade Portuguesa de Cuidados Intensivos para a Abordagem da COVID-19 em Medicina Intensiva ${ }^{5}$;

- antibioterapia sempre que existia a possibilidade de sobreinfeção bacteriana;

- anticoagulação em dose profilática ou terapêutica nos casos de forte suspeita ou tromboembolismo pulmonar confirmado.
Nenhum doente recebeu os fármacos antivirais, antimaláricos, mucolíticos ou imunomoduladores preconizados inicialmente na primeira fase da pandemia COVID-19, uma vez que, atualmente, não existe benefício terapêutico comprovado e bem documentado.6,7

\section{RESULTADOS}

Durante este período (1 de fevereiro a 3 de março), foram seguidos na UVI 26 doentes, de um total de 28 internamentos (2 doentes corresponderam a reinternamento) sendo os indicadores registados em processo clínico eletrónico e na base de dados criada pela equipa.

Verificou-se um predomínio do sexo masculino (69\%), com idades compreendidas entre os 53 anos e os 98 anos (idade média de 76,8 anos). O grupo etário maioritário, 11 doentes (42,3\%), estava compreendido entre os 71 e os 80 anos, seguido de seis doentes (23\%) entre os 81 e os 90 anos. De realçar, ainda, que cinco doentes se encontravam entre os 53 e os 60 anos, dois doentes entre os 91 e os 98 anos, e os restantes dois entre os 61 e os 70 anos.

A maioria dos internamentos teve origem no atendimento permanente (50\%), nove casos foram provenientes da enfermaria COVID (34,6\%), e quatro foram enviados pela UCIP após ventilação invasiva e com necessidade de vigilância intensiva, sob terapêutica com VNI e/ou HFNO.

Relativamente às alterações radiológicas, detetadas à data de admissão na UVI em tomografia computorizada (TC) de tórax (um doente efetuou apenas radiografia do tórax), verificou-se maioritariamente uma apresentação imagiológica em que predominou o vidro despolido em 22 doentes (85\%), na sua maioria com envolvimento de mais de 75\% do parênquima, associado a condensação parenquimatosa em sete e padrão "crazy paving" em cinco; apenas dois doentes apresentavam condensação parenquimatosa sem outras alterações.

Em todos os doentes, o motivo de admissão foi insuficiência respiratória hipoxémica e, à data de entrada, foi necessário instituir HFNO e/ou VNI em 23 doentes. Foram submetidos a HFNO 17 doentes (65,4\%), a CPAP 16 doentes (em dois CPAP com helmet) (61,5\%), e em 10 foram utilizadas as duas terapias respiratórias alternadamente. Em três doentes (11,5\%) foi apenas administrada oxigenoterapia por máscara de Venturi.

No total dos doentes internados na UVI, 22 (85\%) apresentavam, pelo menos, uma comorbilidade, e 15 (58\%) apresentavam mais de uma comorbilidade: hipertensão arterial (15), doença cardíaca (13), obesidade com índi- 
ce de massa corporal $>30 \mathrm{~kg} / \mathrm{m}^{2}$ (9), doença respiratória (8), diabetes (4), doença neurológica (4), hipotiroidismo (4), doença neoplásica (4) e doença renal crónica (2). Apenas quatro doentes (15\%) não apresentavam comorbilidades.

A duração média do internamento na UVI foi de 6,2 dias (mínimo de 1 dia em três doentes e máximo de 21 dias).

Relativamente à terapêutica, a corticoterapia foi instituída (ou mantida) em 24 doentes (18 sob dexametasona, e seis sob metilprednisolona), a antibioterapia em 22 doentes (85\%), por suspeita ou confirmação de sobreinfeção bacteriana - ceftriaxona+azitromicina (em 11 doentes) e piperacilina/tazobactam (em 11 doentes) foram as associações mais frequentes, estando algumas já em curso aquando da receção dos doentes na UVI; amoxicilina+ácido clavulânico em três doentes e meropenem em dois doentes -, e a anticoagulação em dose terapêutica em quatro doentes (15\%), por tromboembolismo confirmado. Todos os outros doentes receberam anticoagulação profilática. Três doentes apresentaram um quadro de delírio com necessidade de neurolépticos, e um doente desenvolveu pneumomediastino/pneumotórax iatrogénico (sem necessidade de drenagem), associado a CPAP com helmet.

No que concerne à evolução clínica, cinco doentes foram transferidos para a UCIP por agravamento e necessidade de ventilação invasiva, 11 doentes foram transferidos para o internamento em Medicina por já não necessitarem de VNI ou HFNO, um doente foi transferido para o hospital de residência a pedido dos familiares, cinco doentes tiveram alta diretamente para o domicílio, e quatro doentes faleceram na UVI, com as seguintes idades e comorbilidades: 98 anos (1 dia de internamento e hipertensão arterial-HTA, insuficiência cardíaca e doença pulmonar obstrutiva crónica-DPOC), 88 anos (6 dias de internamento e obesidade, diabetes, doença cardíaca e hipotiroidismo), 83 anos (20 dias de internamento e doença de Parkinson, DPOC, HTA e hipotiroidismo), e 78 anos (21 dias de internamento e Alzheimer com 6 anos de evolução).

Dos doentes transferidos, um faleceu em enfermaria (92 anos com antecedentes de demência, insuficiência cardíaca e HTA) e quatro vieram a falecer na UCIP tendo as seguintes idades e comorbilidades: 84 anos com doença cardíaca, doença neoplásica e HTA; 76 anos e HTA; 72 anos com antecedentes de neoplasia, enfisema pulmonar e HTA; 53 anos e obesidade.

Assim, no total dos 26 doentes que passaram pela UVI, 16 tiveram alta hospitalar (62\%), nove faleceram (35\%), e um doente ainda se encontra internado à data de redação deste artigo.

\section{DISCUSSÃO}

A pandemia COVID-19 provocou, a nível global, uma pressão sem precedentes no sistema de saúde.

Realça-se a plasticidade organizativa demonstrada pelo Hospital CUF Tejo, a coordenação do trabalho desenvolvido com a Administração, a cooperação e articulação com as equipas do atendimento permanente, da medicina interna e da unidade de cuidados intensivos.

Antigamente, o saber era mais curto e, por isso, mais limitada a ignorância, pois apenas quando se sabe muito, se percebe a amplitude do que se ignora. ${ }^{8}$

Sabíamos que era fundamental a monitorização contínua, a avaliação em curtos intervalos, e que a taxa de sucesso publicada pelas unidades de cuidados intermédios com as melhores séries, tinha em consideração doentes corretamente selecionados, profissionais bem treinados e equipamentos adequados disponíveis. ${ }^{4}$

Tínhamos consciência de que, quanto mais cedo fossem aplicadas as terapêuticas respiratórias não invasivas (nas fases iniciais de ARDS induzido pela COVID-19 nos doentes em respiração espontânea em que a mecânica pulmonar ainda está preservada e a doença vascular tromboembólica tem menos probabilidade de se ter desenvolvido), maior probabilidade de sucesso estas teriam de evitar a intubação. ${ }^{4}$

Percebíamos, também, que prolongar a NRIT na ausência de melhoria clínica/gasométrica exporia o doente ao risco de atraso na intubação, com aumento da mortalidade. Finalmente, o objetivo da UVI era também o de preservar a capacidade de resposta dos cuidados intensivos, local onde residia a admissão dos doentes mais graves.

Preocupávamo-nos o facto de os profissionais da UVI não terem, na sua maioria, experiência prévia ou recente em cuidados intermédios, e terem de adquirir formação acelerada com a "máquina já em andamento". No entanto, a vontade de fazer bem, o empenho, a solidariedade, a energia e o entusiasmo de todos, foram determinantes para, rapidamente, toda a equipa se preparar para a tarefa que nos foi destinada.

Na prática clínica, havia uma grande diversidade na abordagem terapêutica da insuficiência respiratória aguda relacionada com a COVID-19, em virtude de não existir evidência e consenso sobre o tratamento ideal.9 
As terapêuticas com HFNO e VNI foram inicialmente evitadas devido à preocupação de elevada geração de aerossóis, e na convicção de que estes doentes rapidamente se deterioravam e necessitavam de ventilação mecânica. ${ }^{10}$ No entanto, a experiência favorável em pequenos estudos e o aumento da disponibilidade de EPI levou a um aumento na utilização de terapias respiratórias não invasivas. ${ }^{11,12}$

A falência respiratória aguda relacionada com a COVID-19 tem vindo a ser tratada com sucesso por terapêuticas respiratórias não invasivas (HFNO, CPAP ou BIPAP), evitando a intubação traqueal e a ventilação invasiva. Recentes publicações (total de 21, incluindo 1553 doentes), revistas no artigo de Winck JC e Scali R, referem taxas de sucesso com HFNO, CPAP e BPAP de 60\%, 55\% e 59\%, respetivamente. ${ }^{4}$ A heterogeneidade dos protocolos implementados das TRNI é significativa. $\bigcirc$ trabalho mencionado, baseado na experiência com TRNI na insuficiência respiratória aguda induzida pela COVID-19, propõe um algoritmo com decisões de quando iniciar, suspender e desmame das diferentes terapias respiratórias não invasivas. ${ }^{4}$ Utilizámos na UVI o referido algoritmo, revisitado e simplificado, como ante- riormente referido (Fig. 1).

Atualmente, existe consenso internacional na insuficiência respiratória provocada pela COVID-19, para utilização de oxigénio nasal de alto fluxo ${ }^{13}$ e ventilação não invasiva, associada ao decúbito ventral para melhorar a oxigenação e potencialmente reduzir a intubação traqueal. ${ }^{14}$

Estes doentes, com suporte respiratório não invasivo, precisam de acompanhamento contínuo, para evitar atrasos na intubação traqueal, particularmente indicada na alteração de estado de consciência, instabilidade hemodinâmica, hipoxemia grave, aumento de hipercapnia e/ou aumento da frequência respiratória. Os critérios clínicos na decisão de intubação traqueal constituem fator determinante na tomada de decisão. ${ }^{15}$ Muitos peritos advogam a "early intubation", mas não é clara a definição de "early". A transição para os cuidados intensivos pode ser rápida, o que exige uma permanente comunicação e informação dos doentes sob terapêutica não invasiva, os quais podem rapidamente ter necessidade de transferência para ventilação mecânica. Os cinco doentes transferidos para os cuidados intensivos tiveram a avaliação conjunta das equipas da UVI e UCIP.

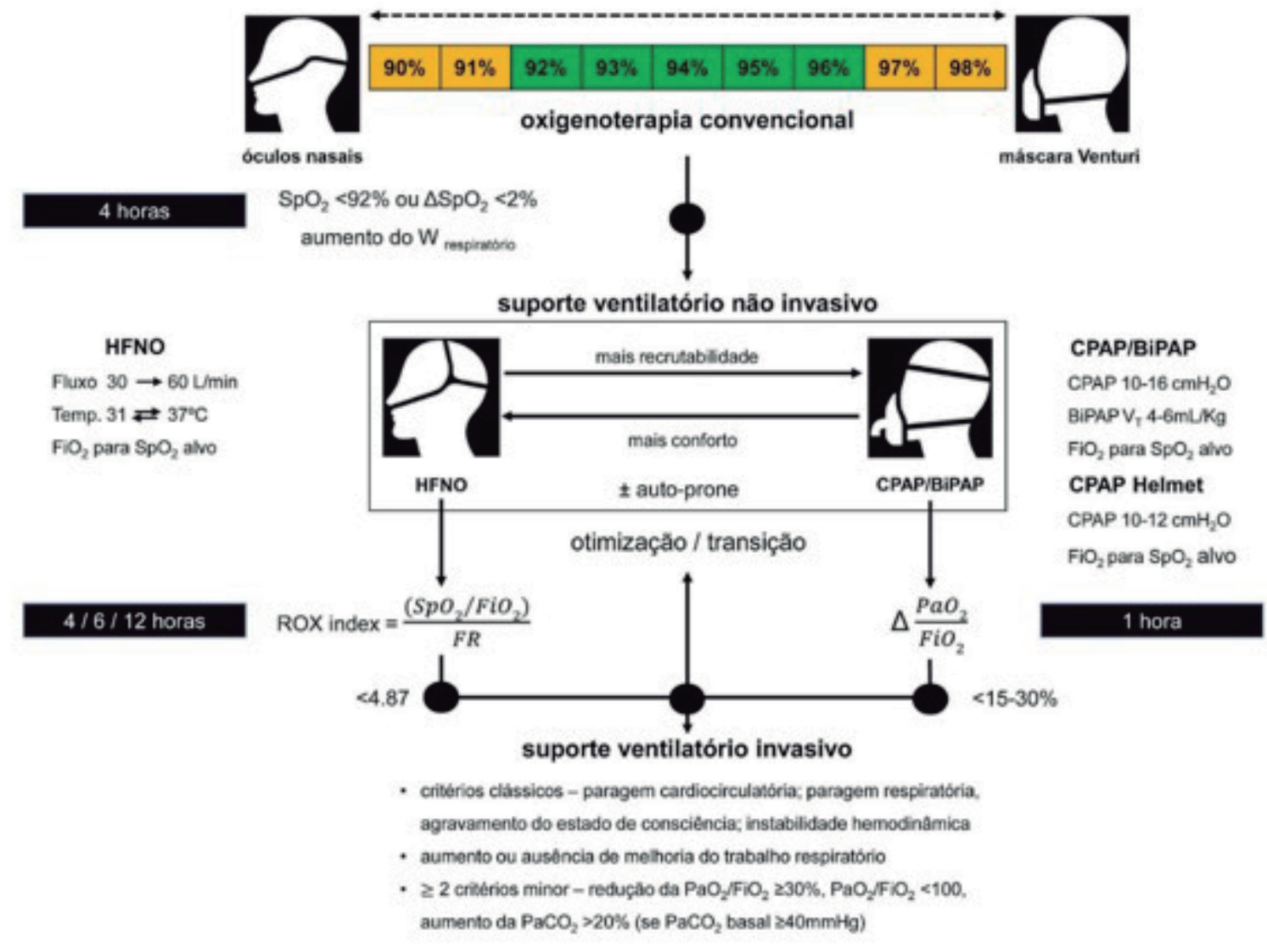

FIGURA 1. Algoritmo TRNI na insuficiência respiratória aguda induzida pela COVID-19 (autor: Dr. João João Mendes). 
Existe, também, consenso internacional na utilização de corticoterapia sistémica, que deve ser utilizada nos doentes internados com COVID-19 que necessitam de oxigénio suplementar $\left(\mathrm{SpO}_{2}<94 \%\right.$ e/ou $\left.\mathrm{PaO}_{2} / \mathrm{FiO}_{2}<300\right)$. A eficácia foi demonstrada, sendo a dexametasona a preferida (dose diária de 6 mg e duração preferencial de 5 a 10 dias). ${ }^{16,17}$

A infeção por SARS-CoV-2 tem sido associada a um aumento do risco de tromboembolismo venoso (TEV) atribuído a características de hipercoagulabilidade, devido ao estado hiperinflamatório desencadeado pela resposta imune do hospedeiro contra o SARS-CoV-2, e caracterizado pelo aumento dos níveis de D-dímero. ${ }^{18}$ A incidência de TEV é altamente variável. Estimativas recentes, numa revisão de 48 estudos foram, de 17\% para TVE, 7,1\% para embolia pulmonar e 12,1\% para a trombose venosa profunda. ${ }^{19}$ Na nossa casuística tivemos quatro casos (15\%) de tromboembolismo pulmonar confirmado.

Não existe, ainda, uma recomendação consensual sobre a dose de anticoagulação recomendada (profilática, alta dose profilática ou terapêutica) nos doentes hospitalizados com COVID-19, apesar de, em algumas situações, se sugerir profilaxia com dose intermédia.,20,21

A coinfeção bacteriana nos doentes COVID-19 é referida com pouca frequência, tendo sido isolado o agente patogénico bacteriano em $<10 \%$ dos doentes. ${ }^{22}$ TrabaIhos publicados sobre infeções bacterianas em doentes COVID-19 hospitalizados referem coinfeção bacteriana em 3,5\%, e ocorrência de infeções bacterianas secundárias durante o internamento até 15\%.22 Na prática clínica, não é fácil confirmar se existe infeção bacteriana secundária associada no doente internado por COVID-19. Na doença grave, os parâmetros inflamatórios, procalcitonina e outros, estão frequentemente elevados, mas são os critérios clínicos que devem estar na ponderação de iniciar ou suspender a antibioterapia, ${ }^{23}$ que deve ser instituída na presunção de sobreinfeção bacteriana - e foi essa a nossa decisão em 85\% dos doentes. Recentes normas de orientação clínica publicadas recomendam a instituição de antibioterapia empírica nos doentes graves com COVID-19, apesar da maioria dos casos poderem não necessitar de antibioterapia empírica. ${ }^{11,24}$

A insuficiência respiratória aguda induzida pela COVID-19 tem uma apresentação heterogénea, e é debatida a presença de fenótipos específicos. ${ }^{25,26} \mathrm{~A}$ apresentação imagiológica inicial mais comum consiste na presença de parênquima pulmonar com vidro despolido, mas a evolução é variável de acordo com o demonstrado na nossa série. Ilustramos a evolução imagiológica de um doente que - tendo vindo a falecer na UCIP num to- tal de 26 dias de internamento (10 dias na UVI e 16 dias na UCIP), apresentando apenas como comorbilidade HTA - exemplifica a variabilidade, diferente expressão e evolução clínica desta doença. À entrada, a TC torácica apresentava apenas vidro despolido exuberante (Fig. 2A) e, 25 dias depois, sem resposta às terapêuticas utilizadas, e apesar de "lung-protective ventilation", verificou-se um franco agravamento imagiológico com extensas áreas em vidro despolido associadas a lesões quísticas (Fig. 2B), englobadas no espectro de lesão pulmonar associada ao ventilador, similares às encontradas noutros doentes e já anteriormente publicadas. ${ }^{27}$

Os doentes que faleceram na UVI correspondiam ao "teto terapêutico" definido pela equipa médica, não sendo de escalar para ventilação invasiva (familiares informados e em sintonia da decisão de não intubar).

Os familiares dos doentes internados na UVI, privados da visita aos mesmos durante a sua estadia na unidade, foram contactados diariamente pela equipa médica, fornecendo informações sobre a situação clínica dos pacientes.

De realçar que não se verificaram casos de infeção por SARS-CoV-2 entre os profissionais de saúde.

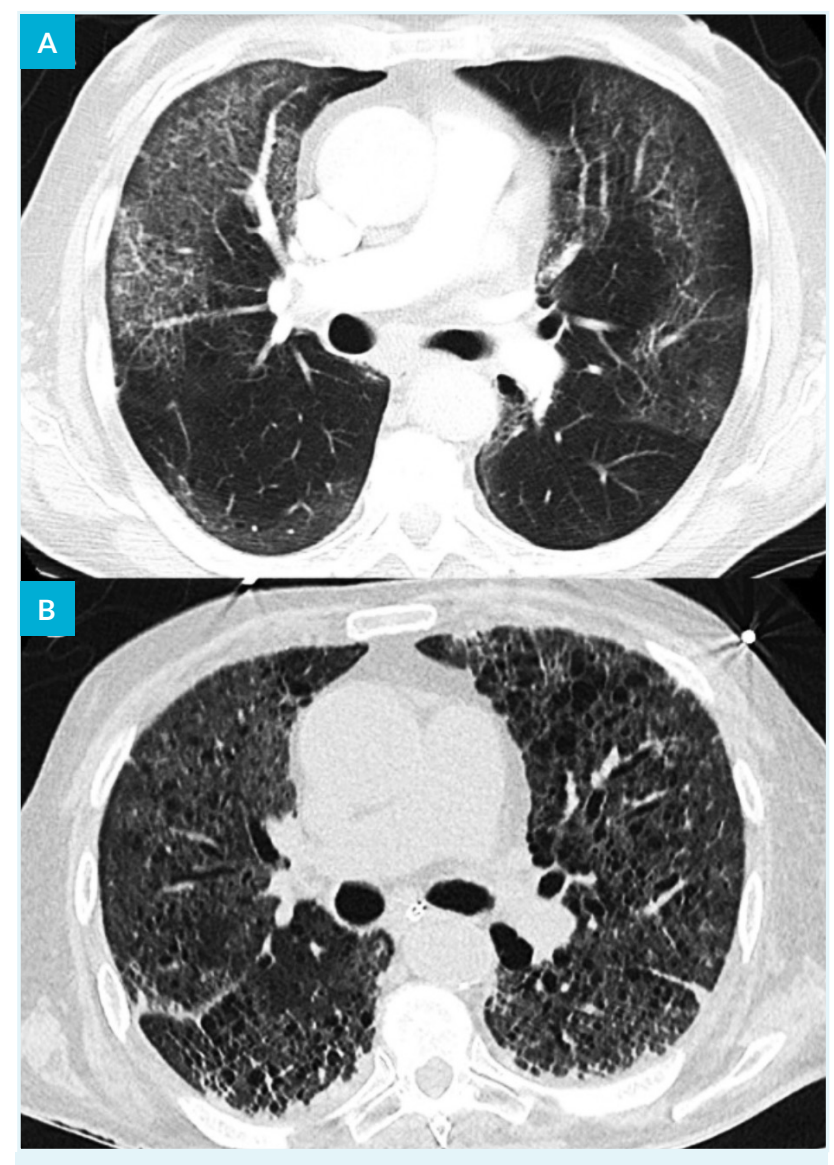

FIGURA 2A e 2B. TC torácica inicial com vidro despolido multilobar (A) e TC torácica de reavaliação 4 semanas depois com vidro despolido, distorção da arquitetura pulmonar e presença de lesões quísticas bilaterais $(\mathrm{B})$. 


\section{CONCLUSÃO}

Não é objetivo deste artigo retirar ilações científicas sobre as terapias respiratórias não invasivas na insuficiência respiratória aguda relacionada com a COVID-19, mas sim relatar a nossa experiência e as bases científicas que estiveram na génese da nossa atuação.

Recentemente, diversas sociedades científicas publicaram atualizações sobre a abordagem e o tratamento de doentes internados por COVID-19 e insuficiência respiratória aguda. 714,28

Seguramente que, num futuro próximo, novas publicações trarão clarividência sobre áreas em que ainda residem algumas incertezas no tratamento da COVID-19. No dia 3 de março, o número de doentes internados tornou dispensável a estrutura da UVI, e regressámos com energia e entusiasmo renovado ao exercício habitual da nossa atividade profissional, funções que acumulámos com o trabalho suplementar da UVI.

A frase de Joshua Lederberg tem mais de meio século, e o futuro continuou a dar-Ihe razão: muitos milhares de pessoas foram já vencidas pelos mais pequenos seres que povoam o nosso planeta - as bactérias e os vírus.

Como serão os próximos capítulos? Ninguém sabe a resposta.

"We live in evolutionary competition with microbes - bacteria and viruses. There is no guarantee that we will be the survivors." 29

Muitas pessoas consideram que a COVID-19 marca o início de novas pandemias.

Por nós, gostaríamos que esta história não tivesse mais capítulos e fosse apenas mais uma, de tantas outras (ultra)passadas, nos registos da nossa "memory lane".

\section{AGRADECIMENTOS/ ACKNOWLEDGMENTS}

Não teria sido possível construir a infraestrutura da UVI sem contar com a experiência e conselhos imprescindíveis de quem há muito exerce atividade em cuidados Intensivos - Dr. João João Mendes e Dr. Pedro Ponce.

Um agradecimento especial aos médicos que asseguraram as escalas da UVI. Rapidamente e sem reservas se disponibilizaram, permitindo assegurar uma vigilância adequada dos doentes - Dr. Luís Heitor, Dr. Mário Ferreira, Dr. Nuno Brito e Dr. Ricardo Taborda.

Um agradecimento aos Enfermeiros João Branco, Sónia
Santos e Bárbara Freitas, muito importantes na organização da UVI.

A todos os enfermeiros/enfermeiras, fisioterapeutas e auxiliares de ação médica que prestaram serviço e foram vitais para o desempenho da UVI.

À Administração do Hospital (Dra. Rita Costa e Dr. Gonçalo Marcelino) e à Direção de Enfermagem (Enfermeiro José Coelho), incansáveis no apoio e procura de soluções.

\section{RESPONSABILIDADES ÉTICAS}

CONFLITOS DE INTERESSE: Os autores declaram a inexistência de conflitos de interesse na realização do presente trabalho.

FONTES DE FINANCIAMENTO: Não existiram fontes externas de financiamento para a realização deste artigo.

CONFIDENCIALIDADE DOS DADOS: Os autores declaram ter seguido os protocolos da sua instituição acerca da publicação dos dados de doentes.

PROTEÇÃO DE PESSOAS E ANIMAIS: Os autores declaram que os procedimentos seguidos estavam de acordo com os regulamentos estabelecidos pelos responsáveis da Comissão de Investigação Clínica e Ética e de acordo com a Declaração de Helsínquia da Associação Médica Mundial.

PROVENIÊNCIA E REVISÃO POR PARES: Não comissionado; revisão externa por pares.

\section{ETHICAL DISCLOSURES}

CONFLICTS OF INTEREST: The authors have no conflicts of interest to declare.

FINANCING SUPPORT: This work has not received any contribution, grant or scholarship.

CONFIDENTIALITY OF DATA: The authors declare that they have followed the protocols of their work center on the publication of data from patients.

PROTECTION OF HUMAN AND ANIMAL SUBJECTS: The authors declare that the procedures followed were in accordance with the regulations of the relevant clinical research ethics committee and with those of the Code of Ethics of the World Medical Association (Declaration of Helsinki).

PROVENANCE AND PEER REVIEW: Not commissioned; externally peer reviewed. 


\section{REFERÊNCIAS}

1. CUF. Resposta da rede Cuf no combate à pandemia COVID-19, comunicado interno Comissão Executiva, publicado a 17 de março de 2020 [acedido em março de 2021] Disponível em: http://www.cuf.pt.

2. CUF. COVID-19, Boletim informativo \#2 CUF. [acedido em março de 2021] Disponível em: http://www.cuf.pt.

3. Herrera $\vee$, Finkler N, Vincent J. Innovation and transformation in the response to COVID-19: seven areas where clinicians need to lead. NEJM Catalyst Innov Care Deliv. 2020;16:10.1056/CAT.20.0087. doi: 10.1056/CAT.20.0087.

4. Winck JC, Scala R. Non-invasive respiratory support paths in hospitalized patients with COVID-19: proposal of an algorithm. Pulmonology. 2021:20; S2531-0437(20)30265-8. doi: 10.1016/j.pulmoe.2020.12.005.

5. Mendes JJ, Mergulhão P, Paiva JA, Gouveia J. Actualização das Recomendações COVID-19 2020. [acedido em março de 2021] Disponível em: https://www.spci.pt/media/COVID-19/ Recomendacoes_SPCI_oxigenoterapia_suporte_V2.pdf.

6. National Institute of Health (NIH). COVID-19, Treatment Guidelines 2020 [acedido em março de 2021] Disponível em: https://www.covid19treatment-guideleines.nih.gov/overview/.

7. Chalmers JD, Crichton ML, Goeminne PC, Cao B, Humbert M, Shteinberg $\mathrm{M}$, et al. Management of hospitalised adults with coronavirus disease-19 (COVID-19): A European Respiratory Society living guideline. Eur Respir J. 2021:2100048. doi: 10.1183/13993003.00048-2021.

8. Lobo Antunes J. O Eco Silencioso. Lisboa: Gradiva; 2008.

9. Azoulay E, de Waele J, Ferrer R, Staudinger T, Borkowska $\mathrm{M}$, Povoa P, et al. International variation in the management of severe COVID-19 patients. Crit Care. 2020;24:486. doi: 10.1186/s13054-020-03194-w.

10. Coronavirus disease 2019 (COVID-19): Critical care and airway management issues. [acedido em março de 2021] Disponível em: https://uptodate.com.

11. NHS England. Guidance for the role and use of non-invasive respiratory support in adult patients with COVID19 (confirmed or suspected) 6 April 2020, Version 3. [acedido em março de 2021] Disponível em: https://www.england.nhs.uk/coronavirus/wp-content/ uploads/sites/52/2020/03/specialty-guide-NIV-respiratory-support-and-coronavirus-v3.pdf.

12. Alhazzani W, Møller MH, Arabi YM, Loeb M, Gong MN, Fan E, et al. Surviving sepsis campaign: guidelines on the management of critically ill adults with Coronavirus Disease 2019 (COVID-19). Intensive Care Med. 2020;46:854-87.

13. Rochwerg B, Einav S, Chaudhuri D, Mancebo J, Mauri T, Helviz $Y$, et al. The role for high flow nasal cannula as a respiratory support strategy in adults: a clinical practice guideline. Intensive Care Med. 2020;46:2226-37.

14. Nasa P, Azoulay E, Khanna AK, Jain R, Gupta S, Javeri Y, et al. Expert consensus statements for the management of COVID-19-related acute respiratory failure using a Delphi method. Crit Care. 2021;25:106. doi: 10.1186/s13054-021-03491-y.

15. Wei H, Jiang B, Behringer EC, Hofmeyr R, Myatra SN, Wong DT, et al. Controversies in airway management of COVID-19 patients: updated information and international expert consensus recommendations. Br J Anaesth. 2021;126:361-6.

16. World Health Organization. COVID-19 clinical management: living guidance COVID-19. Living guidance. Updated $25 \mathrm{Ja}$ nuary 2021. [acedido em março de 2021] Disponível em: https://apps.who.int/iris/handl e/10665/338882.

17. RECOVERY Collaborative Group, Horby P, Lim WS, Emberson JR, Mafham M, Bell JL, et al. Dexamethasone in Hospitalized
Patients with Covid-19. N Engl J Med. 2021;384:693-704. doi: 10.1056/NEJMoa2021436.

18. Mouhat B, Besutti M, Bouiller K, Grillet F, Monnin C, Ecarnot F, et al. Elevated D-dimers and lack of anticoagulation predict PE in severe COVID-19 patients. Eur Respir J. 2020;56:2001811. doi: 10.1183/13993003.01811-2020.

19. Jiménez D, García-Sanchez A, Rali P, Muriel A, Bikdeli B, Ruiz-Artacho P, et al. Incidence of VTE and Bleeding Among Hospitalized Patients With Coronavirus Disease 2019: A Systematic Review and Meta-analysis. Chest. 2021;159:1182-96. doi: 10.1016/j.chest.2020.11.005.

20. Moores LK, Tritschler T, Brosnahan S, Carrier M, Collen JF, Doerschug K, et al. Prevention, Diagnosis, and Treatment of VTE in Patients with Coronavirus Disease 2019: CHEST Guideline and Expert Panel Report. Chest. 2020;158:1143-63. doi: 10.1016/j.chest.2020.05.559.

21. Sociedade Portuguesa de Medicina Interna, Núcleo de Estudos de Doença Vascular pulmonar. Recomendações - Prevenção e Tratamento do Tromboembolismo Venoso em Doentes com COVID-19. [acedido em abril de 2021] Disponível em: https://www.spmi.pt/prevencao-e-tratamento-do-tromboembolismo-venoso-em-doentes-com-covid-19/.

22. Langford BJ, So M, Raybardhan S, Leung V, Westwood D, MacFadden DR, et al. Bacterial co-infection and secondary infection in patients with COVID-19: a living rapid review and meta-analysis. Clin Microbiol Infect. 2020;26:1622-9. doi: 10.1016/j.cmi.2020.07.016.

23. Metlay JP, Waterer GW, Long AC, Anzueto A, Brozek J, Crothers $\mathrm{K}$, et al. Diagnosis and treatment of adults with community-acquired pneumonia. An official clinical practice guideline of the American Thoracic Society and Infectious Diseases Society of America, Am J Respir Crit Care Med. 2019;200:e45-e67. doi: 10.1164/rccm.201908-1581'T.

24. Sieswerda E, de Boer MG, Bonten MM, Boersma WG, Jonkers RE, Kullberg BJ, et al, Recommendations for antibacterial therapy in adults with COVID-19 - an evidence-based guideline. Clin Microbiol Infect. 2021;27:61-6. doi: 10.1016/j. cmi.2020.09.041.

25. Rajendram R. Building the house of CARDS by phenotyping on the fly. Eur Respir J. 2020;56:2002429. doi: 10.1183/13993003.02429-2020.

26. Bos LDJ, Sinha P, Dickson RP. The perils of premature phenotyping in COVID-19: a call for caution. Eur Respir J. 2020;56:2001768. doi: 10.1183/13993003.01768-2020.

27. Gupta VK, Alkandari BM, Mohammed W, Tobar AM, Abdelmohsen $\mathrm{AH}$. Ventilator associated lung injury in severe COVID-19 pneumonia patients - Case Reports: Ventilator associated lung injury in COVID-19. Eur J Radiol Open. 2021;8:100310. doi: 10.1016/j.ejro.2020.100310.

28. COVID-19 Treatment Guidelines Panel. Coronavirus Disease 2019 (COVID-19) Treatment Guidelines. National Institutes of Health. [acedido em abril de 2021] Disponível em: https:// www.covid19treatmentguidelines.nih.gov.

29. Lederberg J. Emerging viruses, emerging threat. Science. 1990;247:279-80. doi: 10.1126/science.2153314. 